\title{
On the Geometric Structure of Minimal Dilations on Hilbert $C^{*}$-Modules
}

\author{
D. Popovici
}

\begin{abstract}
We build a unitary extension for an isometry on a Hilbert $C^{\bullet}$-module and then, with this extension help, we obtain the minimal unitary dilation for an adjointable contraction starting from one of its isometric dilations. Having as a starting point a result of B. Sz.-Nagy and $\mathrm{C}$. Foias regarding the geometric structure of the minimal unitary dilations for Hilbert space contractions we prove that this structure maintains itself on Hilbert modules. Finally, we present a necessary and sufficient condition on the minimal isometric dilation in order to admits a Wold-type decomposition, condition which also assures the complementability of the residual part space of the minimal unitary dilation.
\end{abstract}

Keywords: Hilbert $C^{\bullet}$-modules, adjointable contractions, Wold-type decompositions, isometric and unitary dilations

AMS subject classification: $47 \mathrm{C} 15,47$ A 20

\section{Introduction}

For a contraction $T$ on a Hilbert space $\mathcal{H}$ B. Sz.-Nagy proved in 1953 [14] the existence of a minimal unitary dilation $U$ on a certain Hilbert space $\mathcal{K} \supset \mathcal{H}$, both $\mathcal{K}$ and $U$ being unique up to an isomorphism. Two years later J. J. Schäffer gave in [11] a short matrix proof which permitted the study of many applications. This extremely fertile domain has incited the interest of mathematicians all over the world. Therefore, the minimal isometric and unitary dilations for a Hilbert space contraction, their geometric structure and the functional model with the help of the characteristic function played an important role in the spectacular progress made in the understanding of the spectral theory of non-selfadjoint operators on Hilbert spaces. The series of results obtained by B. Sz.-Nagy and C. Foias on Hilbert space contractions, brought together in their famous book "Harmonic Analysis of Operators on Hilbert Space" [15], prove without any doubt the statements above.

Hilbert modules over a $C^{*}$-algebra $A$ were firstly introduced by I. Kaplansky in [3], their main properties being emphasized in the form used today by W. L. Paschke in [6] and M. A. Rieffel in [10]. These objects generalize, in a certain sense, the notion of Hilbert space, but having an $A$-valued inner product. Hilbert modules represent an

D. Popóvici: West Univ. of Timişoara, Dept. Math., Bd. V. Pãrvan 4, 1900 'Timişoara, . Romania; e-mail: danp@tim1.uvt.ro and danp@gaspar2.uvt.ro 
important instrument of study in a general $\mathcal{K}$-theory introduced by $\mathrm{G}$. $\mathrm{G}$. Kasparov and called $\mathcal{K}$-theory, but also in the $C^{*}$-algebraic approach to quantum group theory.

It is our aim, in the present paper, to deal with the properties of adjointable contractions on Hilbert $C^{*}$-modules through their isometric and unitary dilations. Firstly, we build the minimal isometric dilation for such a contraction. Extending this dilation up to a unitary operator we obtain a unitary dilation. The next step will be the study of the geometric structure of these dilations, a special section being dedicated to the residual and *-residual parts of the minimal unitary dilation, because of the important role played be these unitary operators on Hilbert spaces. We could mention here that a Hilbert space contraction $T$ in the class $C_{11}$ is quasi-similar with the residual and *residual parts of its minimal unitary dilation. This result can be used, in connexion with characteristic functions in order to obtain informations about the invariant subspaces of $T$. In the paper [7] (to see [8] also) I have obtained a necessary and sufficient condition on a Hilbert module adjointable isometry in order to admit a Wold-type decomposition, that is to decompose itself in the orthogonal sum between a unitary operator and a unilateral translation. Putting a condition on the adjoint iterates of an adjointable contraction we obtain, as a consequence, a Wold-type decomposition for its minimal isometric dilation.

\section{Notations and preliminaries}

A Hilbert module over a $C^{*}$-algebra $A$ is a right $A$-module $E$ (which also has a linear structure over the complex field, compatible with that of right module) equipped with a linear in the second variable application, $\langle\cdot, \cdot\rangle: E \times E \rightarrow A$ having the following properties:

(P1) $\langle x, x\rangle \geq 0 \quad(x \in E)$, and $\langle x, x\rangle=0$ if and only if $x=0$.

(P2) $\langle x, y a\rangle=\langle x, y\rangle a \quad(x, y \in E, a \in A)$.

(P3) $\langle x, y\rangle^{*}=\langle y, x\rangle \quad(x, y \in E)$.

(P4) $E$ is Banach space with respect to the norm $\|x\|_{E}=\|\langle x, x\rangle\|^{1 / 2} \quad(x \in E)$.

Hilbert spaces are particular cases of Hilbert modules. Other examples of Hilbert modules over the $C^{*}$-algebra $A$ are:

(i) $A$, the inner product being defined by the relation $\langle a, b\rangle=a^{*} b \quad(a, b \in A)$.

(ii) $\ell_{E}^{2}(\mathbb{N})=\left\{\left(x_{n}\right)_{n} \in \prod_{n \in \mathbb{N}} E \mid \sum_{n \in \mathbb{N}}\left\langle x_{n}, x_{n}\right\rangle\right.$ is convergent in norm in $\left.A\right\}$ equipped with the map $\left\langle\left(x_{n}\right)_{n},\left(y_{n}\right)_{n}\right\rangle=\sum_{n \in N}\left\langle x_{n}, y_{n}\right\rangle$ (we shall also use $\ell_{E}^{2}(\mathbb{Z})$ defined in the same way).

A Hilbert submodule $E_{0}$ of a Hilbert A-module $E$ is a closed (with respect to the norm (P4)) submodule of $E$. If $E_{0}$ is a Hilbert submodule of $E$, then we put $E_{0}^{\perp}=\{x \in$ $E \mid\langle x, y\rangle=0$ for all $\left.y \in E_{0}\right\}$. We say that $E_{0}$ is complementable in $E$ if $E=E_{0} \oplus E_{0}^{\perp}$. In general, we write $E_{0} \oplus E_{1}$ instead of $E_{0}+E_{1}$ if $\left\langle E_{0}, E_{1}\right\rangle=0$. If $\left(E_{n}\right)_{n}$ is a sequence of Hilbert submodules of $E$, then

$$
\bigoplus_{n} E_{n}=\left\{x=\sum_{n} x_{n} \mid x_{n} \in E_{n}, \sum_{n}\left(x_{n}, x_{n}\right) \text { is convergent in norm in } A\right\}
$$


is a Hilbert submodule of $E$.

$T: E \rightarrow E$ is said to be adjointable if there exists $T^{*}: E \rightarrow E$ such that $\langle T x, y\rangle=$ $\left\langle x, T^{*} y\right\rangle \quad(x, y \in E)$. Denote by $\mathcal{L}(E)$ the space of all linear and bounded $A$-module maps on $E$ and by $\mathcal{L}_{A}(E)$ its subspace of all adjointable operators. For $T \in \mathcal{L}_{A}(E)$ we use the notation $[E, T]$. An operator $[E, W]$ is called an isometry if $W^{*} W=I_{E}$, and a unitary operator if $W$ and $W^{*}$ are isometries. A necessary and sufficient condition on a linear map $W$ in order to be an adjointable isometry on the Hilbert module $E$ was obtained by E. C. Lance in [4] (to see [9] for an alternative proof): lent:

Theorem 2.1. Let $W$ be a linear map on $E$. The following conditions are equiva-

(i) $W$ is an isometric $A$-module map with complementable range.

(ii) $W$ is adjointable and $W^{*} W=I_{E}$.

An isometry is called a shift or a unilateral translation if there exists a Hilbert submodule $L$ of $E$, wandering for $W$ (that is $\left\langle W^{n} L, L\right\rangle=0$ for $n>0$ ) such that $E=M_{+}(L):=\bigoplus_{n=0}^{\infty} W^{n} L$. If $E=M(L):=\bigoplus_{n \in \mathbf{Z}} W^{n} L$, then $W$ is a unitary operator and is called a bilateral translation. The Wold-type decomposition (obtained in $[7,8])$ mentioned in Section 1 can be synthetized in the following theorem:

Theorem 2.2. An isometry $[E, V]$ admits a Wold-type decomposition $E=E_{0} \oplus E_{1}$ if and only if

$$
\left(\left(V^{* n} x, V^{* n} x\right\rangle\right)_{n} \text { converges in norm in } A, \text { for all } x \in E \text {. }
$$

Furthermore, in the above conditions, $E_{0}=\bigcap_{n>0} V^{n} E$ and $E_{1}=\bigoplus_{n=0}^{\infty} V^{n} L$, where $L=\operatorname{ker} V^{*}$.

One of the consequences that can be deduced from here is a characterization of shift operators: $V$ is a shift if and only if $V^{* n} x \rightarrow 0$, for all $x \in E$.

An adjointable contraction is an operator $[E, T]$ with $\|T\| \leq 1$ (the norm in $\mathcal{L}(E)$ ), or equivalently: $\langle T x, T x\rangle \leq\langle x, x\rangle \quad(x \in E)$. Because $I-T^{*} T$ and $I-T T^{*}$ are positive elements of $\mathcal{L}_{A}(E)$, we can define the defect operators $D_{T}=\left(I-T^{*} T\right)^{1 / 2}$ and $D_{T^{*}}=\left(I-T T^{*}\right)^{1 / 2}$ and the defect modules $\mathcal{D}_{T}=\overline{D_{T} E}$ and $\mathcal{D}_{T^{*}}=\overline{D_{T^{*}} E}$.

We say that $[F, S]$ is a dilation of $[E, T]$ if

$$
E \subset F \quad \text { and } \quad\left\langle T^{n} x, y\right\rangle_{E}=\left\langle S^{n} x, y\right\rangle_{F} \quad(x, y \in E, n \in \mathbb{N}) \text {. }
$$

The notation $\bigvee_{i \in I} E_{i}\left(E_{i}\right.$ being Hilbert submodules of a Hilbert $C^{*}$-module $E$ ) is used for the closure of the submodule generated by all the finite $A$-linear sums with terms in $E_{i}$. The isometric (respectively unitary) dilation $[F, W]$ of the contraction $[E, T]$ is called minimal if $F=\bigvee_{n \geq 0} W^{n} E$ (respectively $\bigvee_{n \in Z} W^{n} E$ ). 


\section{Construction of isometric and unitary dilations}

There exist many ways to build isometric and unitary dilations for an adjointable contraction on a Hilbert $C^{*}$-module. One of these methods is, for example, the one presented by $S$. Itoh in [2] based on the proofs given in [12]. The matrix construction in [11], the abstract extensions on $V H$-spaces in [5] of the dilation theorems obtained by $\mathrm{Sz}$-Nagy can be also used. In this section we apply another method (some ideas can be found in [15]) in order to construct an isometric dilation for an adjointable contraction.

Proposition 3.1. Every isometry $[E, V]$ admits a unitary extension $[F, U]$.

Proof. Let $F=\ell_{E}^{2}(\mathbb{Z})$. Identifying $x \in E$ with $(\ldots, 0, x, 0, \ldots) \in F \quad(x$ is on the position 0 ) we can regard $E$ as a Hilbert submodule of $F$. We build $U: F \rightarrow$ $F, U\left(x_{n}\right)_{n}=\left(y_{n}\right)_{n}$, where $y_{n}=x_{n+1} \quad(n \neq-1,0), y_{-1}=-V^{*} x_{1}$ and $y_{0}=V x_{0}+x_{1}-$ $V V^{*} x_{1}$. With the identifications above $U x=V x(x \in E)$ and so $U$ extends $V$. Further, $U$ has an adjoint $U^{*}: F \rightarrow F, U^{*}\left(y_{n}\right)_{n}=\left(x_{n}\right)_{n}$ where $x_{n}=y_{n-1}(n \neq 0,1), x_{0}=V^{*} y_{0}$ and $x_{1}=-V y_{-1}+y_{0}-V V^{*} y_{0}$. It is simply to observe that $U U^{*}=U^{*} U=I_{F}$ and consequently $[F, U]$ is a unitary extension for $[E, V]$

Corollary 3.2. Let $A$ be a unital $C^{*}$-algebra and $a \in A$ with $a^{*} a=1$. Then there exists a $C^{*}$-algebra $B$ containing $A$ as a $C^{*}$-subalgebra, a unitary $u$ and a projection $p$, elements of $B$, such that $u p=p u p=a$ and $p B p=A$.

Proof. In Proposition 3.1 we take $E=A$ as a Hilbert $A$-module and $F=\ell_{A}^{2}(\mathbb{Z})$. Consider $B=\mathcal{L}_{A}(F)$. The map

$$
A \ni \alpha \longmapsto T_{\alpha} \in B, \quad T_{\alpha}\left(a_{n}\right)_{n}=\left(a_{n}^{\prime}\right)_{n} \quad\left(a_{n}^{\prime}=0(n \neq 0), a_{0}^{\prime}=\alpha a_{0}\right)
$$

is a *-homomorphism of algebras. Since

$$
\left\|T_{\alpha}\right\|^{2}=\sup _{\left\|\left(a_{n}\right)_{n}\right\| \leq 1}\left\|\left(T_{\alpha}\left(a_{n}\right)_{n}, T_{\alpha}\left(a_{n}\right)_{n}\right)\right\|=\sup _{\left\|\left(a_{n}\right)_{n}\right\| \leq 1}\left\|a_{0}^{*} \alpha^{*} \alpha a_{0}\right\|=\left\|\alpha^{*} \alpha\right\|=\|\alpha\|^{2},
$$

the map defined above is also isometric, making possible the identification of $A$ with a $C^{*}$-subalgebra of $B$.

Let $u \in B$ be the unitary operator that extends $a \in A$ defined in Proposition 3.1. Construct $p=T_{1} \in B$ which is a projection (in fact $p$ is the unity of $A$ ). Since $u$ extends $a$ we obtain $u(\alpha)=a \alpha \quad(\alpha \in A)$. Using this observation, a simple calculus shows that $u p=p u p=a$. The equality $p B p=A$ is immediate

Now, let $[E, T]$ be a contraction. We consider the Hilbert $A$-module $F=\ell_{E}^{2}(\mathbb{N})$ and identify $E$ as a Hilbert $A$-submodule of $F$ through the map

$$
E \ni x \longmapsto(x, 0,0, \ldots) \in \ell_{E}^{2}(\mathbb{N}) .
$$

Define $V: F \rightarrow F, V\left(x_{0}, x_{1}, x_{2}, \ldots\right)=\left(T x_{0}, D_{T} x_{0}, x_{1}, x_{2}, \ldots\right)$. The map $V$ is adjointable and, due to the relation $\left\langle T x_{0}, T x_{0}\right\rangle+\left\langle D_{T} x_{0}, D_{T} x_{0}\right\rangle=\left\langle x_{0}, x_{0}\right\rangle$, it is also isometric. Inductively, for $n \in \mathbb{N}$, we obtain

$$
V^{n}\left(x_{0}, x_{1}, \ldots\right)=\left(T^{n} x_{0}, D_{T} T^{n-1} x_{0}, D_{T} T^{n-2} x_{0}, \ldots, D_{T} x_{0}, x_{1}, \ldots\right)
$$


for all $\left(x_{n}\right)_{n} \in F$. Since, for $x, y \in E$ and $n \in \mathbb{N}$,

$$
\left\langle V^{n} x, y\right\rangle=\left\langle\left(T^{n} x, D_{T} T^{n-1} x, \ldots, D_{T} x, 0, \ldots\right),(y, 0, \cdots)\right\rangle=\left\langle T^{n} x, y\right\rangle,
$$

$[F, V]$ is an isometric dilation of $[E, T]$.

To obtain the minimality condition we consider $F_{+}=V_{n \geq 0} V^{n} E$ and denote also with $V$ the restriction to $F_{+}$of the dilation constructed above. For $x, x^{\prime} \in E$ we obtain an orthogonality condition

$$
\left\langle x,\left(V^{n}-T^{n}\right) x^{\prime}\right\rangle=\left\langle x, V^{n} x^{\prime}\right\rangle-\left\langle x, T^{n} x^{\prime}\right\rangle=0 \quad(n \geq 0)
$$

that is

$$
\left\langle E, \bigvee_{n \geq 0}\left(V^{n}-T^{n}\right) E\right\rangle=0
$$

Due to (1) and using the fact that $V^{n} x=T^{n} x+\left(V^{n}-T^{n}\right) x \quad(x \in E, n \geq 0)$ we obtain the orthogonal decomposition

$$
F_{+}=E \oplus \bigvee_{n \geq 0}\left(V^{n}-T^{n}\right) E
$$

With the help of the relations

$$
W x:=T^{*} x \quad \text { and } \quad W\left(V^{n}-T^{n}\right) x:=V^{n-1} x-T^{*} T^{n} x \quad(n \geq 1),
$$

for $x \in E$, we build an $A$-linear map on a dense submodule of $F_{+}$. The fact that $W$ is well-defined and the possibility of extension to $F_{+}$are due to the relation

$$
\begin{aligned}
& \left\langle W\left(\sum_{n}{ }^{\prime}\left(V^{n}-T^{n}\right) x_{n}\right), W\left(\sum_{n}{ }^{\prime}\left(V^{n}-T^{n}\right) x_{n}\right)\right\rangle \\
& \quad \leq\left\langle\sum_{n}{ }^{\prime}\left(V^{n}-T^{n}\right) x_{n}, \sum_{n}^{\prime}\left(V^{n}-T^{n}\right) x_{n}\right\rangle \quad\left(x_{n} \in E\right)
\end{aligned}
$$

(we use the notation $\sum^{\prime}$ only for finite sums). A simple calculation shows that

$$
\begin{aligned}
& \left\langle W\left(\sum_{n}{ }^{\prime}\left(V^{n}-T^{n}\right) x_{n}\right), W\left(\sum_{m}{ }^{\prime}\left(V^{m}-T^{m}\right) x_{m}\right)\right\rangle \\
& \quad=\sum_{m, n}{ }^{\prime}\left(\left\langle V^{n} x_{n}, V^{m} x_{m}\right\rangle-2\left(T^{n} x_{n}, T^{m} x_{m}\right\rangle+\left\langle T^{*} T^{n} x_{n}, T^{*} T^{m} x_{m}\right\rangle\right)
\end{aligned}
$$

and

$$
\begin{aligned}
\left\langle\sum_{n}{ }^{\prime}\left(V^{n}-T^{n}\right) x_{n}, \sum_{m}{ }^{\prime}\left(V^{m}-T^{m}\right) x_{m}\right\rangle \\
=\sum_{m, n}{ }^{\prime}\left(\left\langle V^{n} x_{n}, V^{m} x_{m}\right\rangle-\left\langle T^{n} x_{n}, T^{m} x_{m}\right\rangle\right) .
\end{aligned}
$$

Conșequently, (2) is equivalent to the obvious relation

$$
\left\langle T^{*}\left(\sum_{n}{ }^{\prime} T^{n} x_{n}\right), T^{*}\left(\sum_{n}{ }^{\prime} T^{n} x_{n}\right)\right\rangle \leq\left\langle\sum_{n}{ }^{\prime} T^{n} x_{n}, \sum_{n}{ }^{\prime} T^{n} x_{n}\right\rangle
$$


Furthermore, for $x, x_{n} \in E\left(n \in \mathbb{N}^{*}\right)$ (in a finite number),

$$
\begin{aligned}
\langle W(x & \left.\left.+\sum_{n}{ }^{\prime}\left(V^{n}-T^{n}\right) x_{n}\right), x+\sum_{m}{ }^{\prime}\left(V^{m}-T^{m}\right) x_{m}\right\rangle \\
= & \left\langle T^{*} x+\sum_{n}{ }^{\prime}\left(V^{n-1} x_{n}-T^{*} T^{n} x_{n}\right), x+\sum_{m}{ }^{\prime}\left(V^{m} x_{m}-T^{m} x_{m}\right)\right\rangle \\
= & \langle x, T x\rangle+\sum_{n}{ }^{\prime}\left(\left\langle T^{n-1} x_{n}, x\right\rangle-\left\langle T^{n} x_{n}, T x\right\rangle\right) \\
& +\sum_{m, n}{ }^{\prime}\left(\left\langle V^{n} x_{n}, V^{m+1} x_{m}\right\rangle-\left\langle T^{n-1} x_{n}, T^{m} x_{m}\right\rangle\right)
\end{aligned}
$$

and, by a similar calculus

$$
\left\langle x+\sum_{n}^{\prime}\left(V^{n}-T^{n}\right) x_{n}, V\left(x+\sum_{m}^{\prime}\left(V^{m}-T^{m}\right) x_{m}\right)\right\rangle
$$

has the same value. We have just proved that $V$ has an adjoint and $V^{*}=W$. Obviously, $E$ is invariant to $V^{*}$ and $V^{*} \mid E=T^{*}$.

For the uniqueness let us consider two minimal isometric dilations $\left[F_{+}^{1}, V_{1}\right]$ and $\left[F_{+}^{2}, V_{2}\right]$ for the contraction $[E, T]$. The map

$$
\varphi: F_{+}^{2} \rightarrow F_{+}^{1}, \quad \varphi\left(\sum_{n}{ }^{\prime} V_{2}^{n} x_{n}\right)=\sum_{n}{ }^{\prime} V_{1}^{n} x_{n}
$$

is well-defined and isometric because, for $x, x^{\prime} \in E, m, n \in \mathbb{N}$ and $k=1,2$,

$$
\left\langle V_{k}^{n} x, V_{k}^{n} x^{\prime}\right\rangle= \begin{cases}\left\langle V_{k}^{n-m} x, x^{\prime}\right\rangle=\left\langle T^{n-m} x, x^{\prime}\right\rangle & \text { if } n \geq m \\ \left\langle x, V_{k}^{m-n} x^{\prime}\right\rangle=\left\langle x, T^{m-n} x^{\prime}\right\rangle & \text { if } m>n\end{cases}
$$

and consequently does not depend of the dilation choice. Because the surjectivity is immediate $\varphi$ becomes an isometric $A$-module isomorphism. We say that $\varphi$ is a Hilbert module isomorphism. Furthermore, since $\varphi V_{2}=V_{1} \varphi$ we can say that $\left[F_{+}^{1}, V_{1}\right]$ and $\left[F_{+}^{2}, V_{2}\right]$ are unitary equivalent.

Summarizing those proved above we can enunciate:

Theorem 3.3. Every contraction $[E, T]$ admits a unique (up to a Hilbert module isomorphism) minimal isometric dilation $\left[F_{+}, V\right]$. Furthermore, $E$ is invariant to $V^{*}$ and $V^{*} \mid E=T^{*}$.

Extending the so obtained minimal isometric dilation $\left[F_{+}, V\right]$ to a unitary operator $[F, U]$ (using Proposition 3.1) we find a unitary dilation for the contraction $[E, T]$. The restriction of $U$ to the closed submodule $\bigvee_{n \in \mathbf{Z}} U^{n} E$ then gives a minimal unitary dilation of the contraction $[E, T]$. An argument similar to the one used in the proof of Theorem 3.3 shows the uniqueness, up to a Hilbert module isomorphism, "of the minimal unitary dilation.

We can now formulate:

Theorem 3.4. Every contraction $[E, T]$ admits a minimal unitary dilation. This dilation is unique (up to a Hilbert module isomorphism). 


\section{The geometric structure of isometric and unitary dilation spaces}

In what follows we will always denote by $\left[F_{+}, U_{+}\right]$and $[F, U]$ the minimal isometric and unitary dilations of a given contraction $[E, T]$. In this section we give a detailed description of the geometric structure of Hilbert modules $F_{+}$and $F$. In the case where $E$ is a Hilbert space a similar result is mentioned implicitly in [13] and explicitly in [16]. The decomposition of $F$ obtained below can also serve as a starting point for the dilation construction, as we can see in [1]. We use [15] as a starting point to other results in this section.

In what follows we put

$$
L=\overline{(U-T) E} \quad \text { and } \quad L^{*}=\overline{\left(U^{*}-T^{*}\right) E} .
$$

Recall that $L$ and $L^{*}$ are wandering for $U$ if $\left\langle U^{n} L, L\right\rangle=0$ and $\left\langle U^{n} L^{*}, L^{*}\right\rangle=0$, respectively, for all $n>0$. Recall also from Section 2 that $\mathcal{D}_{T}=\overline{D_{T} E}$ and $\mathcal{D}_{T^{*}}=\overline{D_{T \cdot} E}$ denote the defect modules for $T$.

Proposition 4.1.

(a) $L, L^{*}$ are wandering for $U$.

(b) $L \cong \mathcal{D}_{T}$ and $L^{*} \cong \mathcal{D}_{T} \cdot$ (Hilbert module isomorphisms).

(c) $U L^{*} \oplus U E=E \oplus L=E \vee U E$.

Proof. We prove the properties corresponding to $L$, the other ones being obtained similarly taking into account that $\left[F, U^{*}\right]$ is the minimal unitary dilation of the contraction $\left[E, T^{*}\right]$.

(a) For $x, x^{\prime} \in E$ and $n \in \mathbb{N}^{*}$,

$$
\begin{aligned}
& \left\langle U^{n}(U-T) x,(U-T) x^{\prime}\right\rangle \\
& \quad=\left\langle T^{n} x, x^{\prime}\right\rangle-\left\langle T^{n+1} x, T x^{\prime}\right\rangle-\left\langle T^{n} x, x^{\prime}\right\rangle+\left\langle T^{n+1} x, T x^{\prime}\right\rangle \\
& \quad=0 .
\end{aligned}
$$

By passing to limit it obtains that $L$ is wandering.

(b) $L \ni(U-T) \stackrel{\varphi}{\longrightarrow} D_{T} x \in \mathcal{D}_{T}$ is an isometric, surjective $A$-linear map. Indeed,

$$
\begin{aligned}
\langle(U-T) x,(U-T) x\rangle & =\langle x, x\rangle-\langle T x, T x\rangle-\langle T x, T x\rangle+\langle T x, T x\rangle \\
& =\langle x, x\rangle-\left\langle T^{*} T x, x\right\rangle \\
& =\left\langle D_{T} x, D_{T} x\right\rangle
\end{aligned}
$$

The conclusion follows easily extending $\varphi$ by continuity on $L=\overline{(U-T) E}$.

(c) For the first equality it is sufficient to show that

$$
U\left(U^{*}-T^{*}\right) E \oplus U E=E \oplus(U-T) E
$$


Using the dilation property $\langle U x, y\rangle=\langle T x, y\rangle$ for all $x, y \in E$ one can easily check that the sums are orthogonal. Now if $x^{\prime}, x^{\prime \prime} \in E$ define $x_{1}, x_{2} \in E$ by the equation $\left(\begin{array}{l}x_{1} \\ x_{2}\end{array}\right)=\left(\begin{array}{cc}T^{*} & D_{T}^{2} \\ I & -T\end{array}\right)\left(\begin{array}{c}x^{\prime} \\ x^{\prime \prime}\end{array}\right)$. An easy calculation then shows that

$$
U x_{1}+U\left(U^{*}-T^{*}\right) x_{2}=x^{\prime}+(U-T) x^{\prime \prime} .
$$

Since $\left(\begin{array}{cc}T^{*} & D_{T}^{2} \\ I & -T\end{array}\right)$ is invertible (as operator on $\left.E \oplus E\right)$ with inverse $\left(\begin{array}{cc}T & D_{T^{*}}^{2} \\ I & -T^{*}\end{array}\right)$ this implies the desired equality. The equality $E \oplus L=E \vee U E$ follows from the trivial fact that $E+(U-T) E=E+U E$

For $m, n \geq 0$ and $x, x^{\prime} \in E$

$$
\begin{array}{r}
\left\langle x, U^{n}(U-T) x^{\prime}\right\rangle=\left\langle x, U^{n+1} x^{\prime}\right\rangle-\left\langle x, U^{n} T x^{\prime}\right\rangle=0 \\
\left\langle x, U^{* n}\left(U^{*}-T^{*}\right) x^{\prime}\right\rangle=\left\langle U^{n+1} x, x^{\prime}\right\rangle-\left\langle U^{n} x, T^{*} x^{\prime}\right\rangle=0
\end{array}
$$

and

$$
\begin{aligned}
& \left\langle U^{* n}\left(U^{*}-T^{*}\right) x, U^{m}(U-T) x^{\prime}\right\rangle \\
& \quad=\left\langle x, U^{m+n+2} x^{\prime}\right\rangle-\left\langle x, U^{m+n+1} T x^{\prime}\right\rangle-\left\langle T^{*} x, U^{m+n+1} x^{\prime}\right\rangle+\left\langle T^{*} x, U^{m+n} T x^{\prime}\right\rangle \\
& \quad=0
\end{aligned}
$$

hold. These relations, together with the ones obtained from the fact that the submodules $L$ and $L^{*}$ are wandering for $U$, allow the construction of the Hilbert submodule

$$
F_{0}=\bigoplus_{n \geq 0} U^{* n} L^{*} \oplus E \oplus \bigoplus_{n \geq 0} U^{n} L
$$

As a consequence of the first equality in Proposition $4.1 /(\mathrm{c})$, the relation $U F_{0}=F_{0}$ permits to conclude that $\left[F_{0}, U \mid F_{0}\right]$ is a unitary dilation of $[E, T]$. The relations

$$
\dot{U^{n}} E \subset E \oplus \bigoplus_{k=0}^{n-1} U^{k} L \quad \text { and } \quad U^{* n} E \subset E \oplus \bigoplus_{k=0}^{n-1} U^{* k} L^{*} \quad\left(n \in \mathbb{N}^{*}\right)
$$

obtained by the same equality mentioned above show that $F_{0}=F$.

We are now able to enunciate

Theorem 4.2. Let $[E, T]$ be a contraction and let $[F, U]$ be its minimal unitary dilation. Then

$$
F=\bigoplus_{n \geq 0} U^{* n} L^{*} \oplus E \oplus \bigoplus_{n \geq 0} U^{n} L
$$

Recall from Section 2 that the submodules $M(L)$ and $M_{+}(L)$ of $F$ are defined by $M(L)=\bigoplus_{n \in \mathbf{Z}} U^{n} L$ and $M_{+}(L)=\bigoplus_{n>0} U^{n} L$. Similarly, replacing $L$ by $L^{*}$, we obtain the submodules $M\left(L^{*}\right)$ and $M_{+}\left(L^{*}\right)$. Since $M(L)$ and $M\left(L^{*}\right)$ are clearly invariant for $U$ and $U^{*}$ the same is true for the perpendicular spaces $R=M\left(L^{*}\right)^{\perp}$ and $R_{*}=M(L)^{\perp}$. Thus we can do the following 
Definition 4.3. The unitary operators $\bar{R}=U \mid R$ and $\bar{R} *=U \mid R_{*}$ are called the residual part and the dual (*-residual) part of $U$, respectively.

The fact that, for $x \in E$ and $n \in \mathbb{N}$,

$$
U^{n} x=T^{n} x+(U-T) T^{n-1} x+U(U-T) T^{n-2} x+\cdots+U^{n-1}(U-T) x
$$

leads to the inclusion

$$
F_{+} \subset E \oplus \bigoplus_{n \geq 0} U^{n} L=E \oplus M_{+}(L) .
$$

On the other hand, since

$$
U^{n}(U-T) x=U^{n+1} x-U^{n} T x \in U^{n+1} E \vee U^{n} E \subset F_{+} \quad(x \in E, n \in \mathbb{N})
$$

we obtain $U^{n} L \subset F_{+}$which, together with $E \subset F_{+}$, allow us to prove the converse inclusion $E \oplus M_{+}(L) \subset F_{+}$. Consequently, the minimal isometric dilation space has the geometric structure

$$
F_{+}=E \oplus M_{+}(L)
$$

A simple calculation based on (3) and the equation $U L^{*} \oplus U E=E \oplus L$ of Proposition 4.1 shows that

$$
U_{+} F_{+} \oplus U L^{*}=F_{+} .
$$

As an immediate consequence we obtain the equality $\operatorname{ker} U_{+}^{*}=U L^{*}$. In what follows we will write $L_{*}=U L^{*}\left(=\overline{\left(I-U T^{*}\right) E}\right)$.

We return to the study of the residual and *-residual parts of the minimal unitary dilation $[F, U]$. Applying Theorem 2.2, the minimal isometric dilation $\left[F_{+}, U_{+}\right]$admits a Wold-type decomposition if and only if

$$
\left(\left\langle U_{+}^{* n} y, U_{+}^{* n} y\right\rangle\right)_{n} \quad \text { converges in norm in } A \text {, for all } y \in F_{+} .
$$

Because $U_{+}^{* n} x=T^{* n} x \quad(x \in E)$, (5) leads us to

$$
\left(\left(T^{* n} x, T^{* n} x\right\rangle\right)_{n} \quad \text { converges in norm in } A \text {, for all } x \in \dot{E} \text {. }
$$

Conversely, suppose that (6) holds. If $x, x^{\prime} \in E$ and $m, n \in \mathbb{N}$ with $m<n$, then

$$
\begin{aligned}
&\left\langle U_{+}^{*(n-m)}(U-T) x, U_{+}^{* n}(U-T) x^{\prime}\right\rangle \\
&=\left\langle U_{+}^{*(n-m)} U x, U_{+}^{* n} U x^{\prime}\right\rangle-\left\langle U_{+}^{*(n-m)} U x, U_{+}^{* n} T x^{\prime}\right\rangle \\
&-\left\langle U_{+}^{*(n-m)} T x, U_{+}^{* n} U x^{\prime}\right\rangle+\left\langle U_{+}^{*(n-m)} T x, U_{+}^{* n} T x^{\prime}\right\rangle \\
&=\left\langle U_{+}^{*(n-m-1)} x, U_{+}^{*(n-1)} x^{\prime}\right\rangle-\left\langle U_{+}^{*(n-m-1)} x, U_{+}^{* n} T x^{\prime}\right\rangle \\
&-\left\langle U_{+}^{*(n-m)} T x, U_{+}^{*(n-1)} x^{\prime}\right\rangle+\left\langle U_{+}^{*(n-m)} T x, U_{+}^{* n} T x^{\prime}\right\rangle \\
&=\left\langle T^{*(n-m-1)} x, T^{*(n-m-1)}\left(T^{* m} x^{\prime}\right)\right\rangle-\left\langle T^{*(n-m-1)} x, T^{*(n-m-1)}\left(T^{*(m+1)} T x^{\prime}\right)\right\rangle \\
&-\left\langle T^{*(n-m)}(T x), T^{*(n-m)}\left(T^{*(m+1)} x^{\prime}\right)\right\rangle+\left\langle T^{*(n-m)}(T x), T^{*(n-m)}\left(T^{* m} T x^{\prime}\right)\right\rangle .
\end{aligned}
$$


Furthermore,

$$
\begin{aligned}
& \left\langle U_{+}^{* n} x, U_{+}^{*(n-m)}(U-T) x^{\prime}\right\rangle \\
& \quad=\left\langle T^{* n} x, T^{*(n-m-1)} x^{\prime}\right\rangle-\left\langle T^{* n} x, T^{*(n-m)} T x^{\prime}\right\rangle \\
& \quad=\left\langle T^{*(n-m-1)}\left(T^{*(m+1)} x\right), T^{*(n-m-1)} x^{\prime}\right\rangle-\left\langle T^{*(n-m)}\left(T^{* m} x\right), T^{*(n-m)}\left(T x^{\prime}\right)\right\rangle .
\end{aligned}
$$

Using in (7) the relations

$$
U_{+}^{* n} U^{m}(U-T) x=U_{+}^{*(n-m)}(U-T) x \quad(x \in E ; m, n \in \mathbb{N}, m<n)
$$

we obtain an expression in terms of $T^{*}$ iterates for $\left\langle U_{+}^{* n} y, U_{+}^{* n} y^{\prime}\right\rangle$ with $y$ in $U^{m}(U-T) E$ and $y^{\prime}$ in $U^{m^{\prime}}(U-T) E, m, m^{\prime}<n$. Applying the same relations in (8) we obtain $\left\langle U_{+}^{* n} x, U_{+}^{* n} y\right\rangle$ for $x \in E$ and $y \in U^{m}(U-T) E, m \in \mathbb{N}, m<n$. Because $F_{+}=$ $E \oplus M_{+}(L)$ (equality (3)) we can determine $\left\langle U_{+}^{* n} y, U_{+}^{* n} y\right\rangle$ for $y$ in a dense submodule $F_{+}^{(0)}$ of $F$. The polarization identity and the convergence in the norm of $A$ of the sequence $\left(\left\langle T^{* n} x, T^{* n} x\right\rangle\right)_{n}$, for every $x \in E$ (relation (6)) lead us to the property in (5) for $y$ in $F_{+}^{(0)}$. Because $\left\|U_{+}^{* n}\right\|=1 \quad(n \in \mathbb{N})$, by a standard method we can pass from $F_{+}^{(0)}$ to $F_{+}$. This proves the equivalence of properties (5) and (6).

Using (4) we observe that, for $n \in \mathbb{N}^{*}$,

$$
F_{+}=U_{+} F_{+} \oplus U L^{*}=U_{+}^{2} F_{+} \oplus U L^{*} \oplus U^{2} L^{*}=\ldots=U_{+}^{n} F_{+} \oplus \bigoplus_{k=1}^{n} U^{k} L^{*}
$$

Consequently, every $y \in F_{+}$can be uniquely decomposed in

$$
y=U_{+}^{n} y_{n}+\sum_{k=1}^{n} U^{k} l_{k} \quad\left(y_{n} \in F_{+}, l_{k} \in L^{*}(1 \leq k \leq n)\right)
$$

It is not hard to see that

$$
y=U_{+}^{n} U_{+}^{* n} y+\sum_{k=0}^{n-1} U_{+}^{k}\left(I-U_{+}^{*} U_{+}\right) U_{+}^{* k} y
$$

is the decomposition we are looking for. By (5) the relation

$$
\begin{gathered}
\left\langle U_{+}^{n} U_{+}^{* n} y-U_{+}^{m} U_{+}^{* m} y, U_{+}^{n} U_{+}^{* n} y-U_{+}^{m} U_{+}^{* m} y\right\rangle \\
=\left\langle U_{+}^{* n} y, U_{+}^{* n} y\right\rangle-\left\langle U_{+}^{* m} y, U_{+}^{* m} y\right\rangle
\end{gathered} \quad\left(m, n \in \mathbb{N}, m>n, y \in F_{+}\right)
$$

proves the convergence in $F_{+}$of the sequence $\left(U_{+}^{n} U_{+}^{* n} y\right)_{n}$ to a limit $y_{0}$, element of the Hilbert submodule $\bigcap_{n \geq 0} U_{+}^{n} F_{+}$. Since $\left\langle\bigcap_{n \geq 0} U_{+}^{n} F_{+}, U^{m} L^{*}\right\rangle=0 \quad\left(m \in \mathbb{N}^{*}\right)$ we deduce the geometric structure

$$
F_{+}=\bigcap_{n \geq 0} U_{+}^{n} F_{+} \oplus \bigoplus_{n=1}^{\infty} U^{n} L^{*}
$$


Consequently,

$$
F=F_{+} \oplus \bigoplus_{n=0}^{\infty} U^{* n} L^{*}=\bigcap_{n \geq 0} U_{+}^{n} F_{+} \oplus M\left(L^{*}\right)
$$

that is $M\left(L^{*}\right)$ is complementable in $F$ and $R=M\left(L^{*}\right)^{\perp}=\bigcap_{n \geq 0} U_{+}^{n} F_{+}$.

Suppose now that $M\left(L^{*}\right)$ is complementable in $F$. Then $\bigoplus_{n=1}^{\infty} U^{n} L^{*}$ is complementable in $F_{+}$. Because $\left\langle y, \bigoplus_{k=1}^{\infty} U^{k} L^{*}\right\rangle=0\left(n \in \mathbb{N}^{*}\right)$ is equivalent to $y \in U_{+}^{n} F_{+}(n \in$ $\mathbb{N}^{*}$ ) (by (9)) (that is $\left(\oplus_{n=1}^{\infty} U^{n} L^{*}\right)^{\perp}=n_{n \geq 0} U_{+}^{n} F_{+}$) we obtain (10). It is easy to observe that $(10)$ is a Wold-type decomposition for the isometry $\left[F_{+}, U_{+}\right]$and so, again due to Theorem 2.2, (5) holds. In addition $R=\bigcap_{n \geq 0} U_{+}^{n} F_{+}=\bigcap_{n \geq 0} \vee_{k \geq n} U^{k} E$.

Recall that we denote by $L$, the submodule $U L^{*}$. Considering only orthogonal complements in $E \oplus L$ we have $L^{\perp}=E$ and $L_{*}^{\perp}=U E$. So we can deduce $L^{\perp} \vee L^{\perp}=$ $E \vee U E=E \oplus L$ by the second equality in Proposition 4.1/(c). By passing to the orthogonal complement

$$
L \cap L_{*}=\left(L^{\perp} \vee L_{*}^{\perp}\right)^{\perp}=(E \oplus L)^{\perp}=\{0\}
$$

Concerning the geometric structure of the minimal isometric and unitary dilations we can now formulate

Theorem 4.4. Let $[E, T]$ be a contraction, $\left[F_{+}, U_{+}\right]$be its minimal isometric dilation and $[F, U]$ be its minimal unitary dilation. Then:

(a) $L=\overline{(U-T) E}$ and $L_{*}=\overline{\left(I-U T^{*}\right) E}$ are submodules wandering for $U_{+}$and $U$.

(b) $L \cap L_{*}=\{0\}$.

(c) $F_{+}=E \oplus M_{+}(L)$.

(d) The following assertions are equivalent:

$\left[F_{+}, U_{+}\right]$admits a Wold-type decomposition.

$\left(\left\langle T^{* n} x, T^{* n} x\right\rangle\right)_{n}$ converges in norm in $A$, for all $x \in E$.

$M\left(L_{*}\right)$ is complementable in $F$.

(e) If the equivalent conditions above are true, then

$$
F_{+}=R \oplus M_{+}\left(L_{*}\right), \quad F=R \oplus M\left(L_{*}\right), \quad R=\bigcap_{n \geq 0} U_{+}^{n} F_{+}=\bigcap_{n \geq 0} \bigvee_{k \geq n} U^{k} E
$$

$R$ being the residual part space of $[F, U]$.

\section{Remark 4.5.}

(a) In particular, all the results of Theorem 4.4, as those of the whole paper, maintain on Hilbert spaces. We can mention here that the sequences $\left(\left\langle T^{\bullet n} x, T^{* n} x\right\rangle\right)_{n}(x \in E)$ are convergent in $\mathbb{C}$, being monotonous decreasing positive number sequences.

(b) Replacing the contraction $[E, T]$ with its adjoint $\left[E, T^{*}\right]$ similar proofs show that $M(L)$ is complementable in $F\left(F=R_{*} \oplus M(L)\right)$ if and only if $\left(\left\langle T^{n} x, T^{n} x\right\rangle\right)_{n}$ converges in norm in $A$, for all $x \in E$. In these conditions, for the minimal isometric 
dilation space of the contraction $\left[E, T^{*}\right]$ we shall have the structure $R * \oplus \bigoplus_{n=1}^{\infty} U^{* n} L$, and for the dual part space of $U^{*}, R_{*}=\bigcap_{n \leq 0} \bigvee_{k \leq n} U^{k} E$.

Example 4.6. We can build numerous examples of adjointable contractions $T$ on the Hilbert $A$-module $\ell_{A}^{2}(\mathbb{N})$ for which the convergent condition mentioned in Theorem $4.4 /(\mathrm{d})$ holds. Let $A$ be a unital $C^{*}$-algebra and $a \in A$ with $\|a\| \leq 1$. The map $\left[\ell_{A}^{2}(\mathrm{~N}), T\right]$ defined by

$$
T\left(\left(x_{n}\right)_{n}\right)=\left(\left(1-a^{*} a\right)^{1 / 2} x_{0}-a^{*} x_{1}, a x_{0}+\left(1-a a^{*}\right)^{1 / 2} x_{1}, 0, \ldots\right)
$$

has the adjoint $\left[\ell_{A}^{2}(\mathrm{~N}), T^{*}\right]$,

$$
T^{*}\left(\left(x_{n}\right)_{n}\right)=\left(\left(1-a^{*} a\right)^{1 / 2} x_{0}+a^{*} x_{1},-a x_{0}+\left(1-a a^{*}\right)^{1 / 2} x_{1}, 0, \ldots\right) .
$$

A simple calculus shows that, for all $m \in \mathbb{N}$,

$$
\left\langle T^{* m}\left(\left(x_{n}\right)_{n}\right), T^{* m}\left(\left(x_{n}\right)_{n}\right)\right\rangle=x_{0}^{*} x_{0}+x_{1}^{*} x_{1} .
$$

On the other hand, in contrast with the Hilbert space particular case, not every adjointable contraction $[E, T]$ on a Hilbert module $E$ verifies the condition

$$
\left(\left(T^{* n} x, T^{* n} x\right\rangle\right)_{n} \quad \text { converges in norm in } A \text { for all } x \in E
$$

Indeed, let $A$ be a unital $C^{*}$-algebra and $a \in A$ with $a^{*} a=1$ and $a a^{*} \neq 1$. As we presented in detail in [8], the isometry $\left[A, V_{a}\right], V_{a}(b)=a b(b \in A)$ does not admits a Wold-type decomposition, that is $\left(\left\langle V_{a}^{* n} b, V_{a}^{* n} b\right\rangle\right)_{n}=\left(b^{*} a^{n} a^{* n} b\right)_{n}$ does not converges in norm in $A$ for at least one $b \in A$.

We shall now present some consequences of the Theorem 4.4 keeping the same notations:

Corollary 4.7. If $\left(\left\langle T^{* n} x, T^{* n} x\right\rangle\right)_{n}$ converges in norm in $A$, for all $x \in E$, then the orthogonal projection corresponding to the residual part of $[F, U]$ (its existence is due to the decomposition $F=R \oplus M\left(L^{*}\right)$ ) can be calculated with the help of the formulas

$$
P_{R}\left(U^{m} x\right)=\lim _{n \rightarrow \infty} U^{n} T^{*(n-m)} x \quad(x \in E, m \in \mathbb{N}) .
$$

Furthermore, in the same conditions applied to the contraction $\left[E, T^{*}\right]$, we have

$$
P_{R_{\bullet}}\left(U^{m} x\right)=\lim _{n \rightarrow \infty} U^{* n} T^{n+m} x \quad(x \in E, m \in \mathbb{N}) .
$$

Proof. Denote by $P_{F_{+}}$the othogonal projection on $F_{+}$associated to the decomposition $F=F_{+} \oplus \bigoplus_{n \geq 0} U^{* n} L^{*}$. If $y \in F$, then

$$
\begin{aligned}
y & =P_{F_{+}} y+\left(I_{F}-P_{F_{+}}\right) y \\
& =\lim _{n \rightarrow \infty} U_{+}^{n}\left(U_{+}^{*}\right)^{n} P_{F_{+}} y+\left(P_{F_{+}} y-\lim _{n \rightarrow \infty} U_{+}^{n}\left(U_{+}^{*}\right)^{n} P_{F_{+}} y\right)+\left(I_{F}-P_{F_{+}}\right) y,
\end{aligned}
$$


the last equality being a consequence of the decomposition $F_{+}=R \oplus M_{+}\left(L_{*}\right)$ obtained in Theorem 4.4/(e). Using the equalities $U_{+}^{*} y_{+}=P_{F_{+}} U^{*} y_{+}\left(y_{+} \in F_{+}\right)$we can easily check that

$$
P_{R} y=\lim _{n \rightarrow \infty} U_{+}^{n}\left(U_{+}^{*}\right)^{n} P_{F_{+}} y=\lim _{n \rightarrow \infty} U^{n}\left(U^{*} P_{F_{+}}\right)^{n} y \quad(y \in F)
$$

Because, for $x \in E$ and $m \in \mathbb{Z}$,

$$
U^{m} x= \begin{cases}T^{m} x+(U-T) T^{m-1} x+U(U-T) T^{m-2} x & \\ +\ldots+U^{m-1}(U-T) x & \text { if } m \geq 0 \\ T^{*(-m)} x+\left(U^{*}-T^{*}\right) T^{*(-m-1)} x+U^{*}\left(U^{*}-T^{*}\right) T^{*(-m-2)} x & \\ +\ldots+U^{*(-m-1)}\left(U^{*}-T^{*}\right) x & \text { if } m<0\end{cases}
$$

the formula

$$
P_{F_{+}} U^{m} x= \begin{cases}U^{m} x & \text { if } m \geq 0 \\ T^{*(-m)} x & \text { if } m<0\end{cases}
$$

also holds. Inductively, after $m \in \mathbb{Z}$, it can be shown that

$$
U^{n}\left(U^{*} P_{F_{+}}\right)^{n}\left(U^{m} x\right)=\left\{\begin{array}{ll}
U^{m} x & \text { if } m \geq n-1 \\
U^{n-1} T^{*(n-1-m)} x & \text { if } m \leq n-2
\end{array} \quad(n \in \mathbb{N})\right.
$$

and (11) follows immediately as a consequence

\section{Corollary 4.8 .}

(a) If $T^{* n} x \rightarrow 0$ as $n \rightarrow \infty$, for each $x \in E$, then $R=\{0\}$.

(b) Conversely, if $R=\{0\}$ and $\left(\left\langle T^{* n} x, T^{* n} x\right\rangle\right)_{n}$ converges in norm in $A$, for all $x \in E$, then $T^{* n} x \rightarrow 0$ as $n \rightarrow \infty$, for all $x \in E$. The properties corresponding to the adjoint $\left[E, T^{*}\right]$ also holds.

Proof. (a) Using Corollary 4.7, $\left\|P_{R}\left(U^{m} x\right)\right\|=\lim _{n \rightarrow \infty}\left\|T^{*(n-m)} x\right\|=0$, and so $P_{R}=0$, that is $R=\{0\}$. For statement (b) it is sufficient to observe that $P_{R} x=$ $\lim _{n \rightarrow \infty} U^{n} T^{* n} x=0$, for all $x \in E$ D

\section{References}

[1] Halperin, I.: The unitary dilation of a contraction operator. Duke Math. J. 28 (1961), $563-571$.

[2] Itoh, S.: A note on dilations in modules over $C^{\bullet}$-algebras. J. London Math. Soc. (2) 22 (1980), $117-126$.

[3] Kaplansky, I.: Modules over operator algebras. Amer. J. Math. 75 (1953), $839-858$.

[4] Lance, E. C.: Unitary operators on Hilbert $C^{\bullet}$-modules. Bull. London Math. Soc. 26 (1994), $363-366$.

[5] Loynes, R. M.: On generalized positive-definite functions. Proc. Londoñ Math. Soc. (3) 15 (1965), $373-384$. 
[6] Paschke, W. L.: Inner product modules over $B^{*}$-algebras. Trans. Amer. Math. Soc. 182 (1973), $443-468$.

[7] Popovici, D.: A Wold-type decomposition in Hilbert $C^{\bullet}$-modules. Sem. Lin. Oper. Harm. Anal. (West Univ. of Timişoara) 2 (1996), 1 - 16.

[8] Popovici, D.: Orthogonal decompositions of isometries in Hilbert $C^{*}$-modules. J. Oper. Theory 39 (1998) (to appear).

[9] Popovici, D.: Stationary processes in connection with Wold-type decompositions in Hilbert $C^{*}$-modules. Acta Math. Univ. Comenianae (submitted).

[10] Rieffel, M. A.: Induced representations of $C^{*}$-algebras. Adv. Math. 13 (1974), 176 - 257.

[11] Schäffer, J. j.: On unitary dilations of contractions. Proc. Amer. Math. Soc. 6 (1955), 322.

[12] Stinespring, W. F.: Positive functions on $C^{*}$-algebras. Proc. Amer. Math. Soc. 6 (1955), $211-216$.

[13] Sz.-Nagy, B.: On Schäffer's construction of unitary dilations. Ann. Univ. Budapest, Sect. Math. 3-4 (1960/61), $343-346$.

[14] Sz.-Nagy, B.: Sur les contractions de l'espace de Hilbert. Acta Sci. Math. 15 (1953), 87 $-92$.

[15] Sz.-Nagy, B. and C. Foiaş: Harmonic Analysis of Operators on Hilbert Space. Amsterdam - London: North-Holland Publ. Comp. 1979.

[16] Sz.-Nagy, B. and C. Foiaş: Sur les contractions de l'espace de Hilbert. Part V: Translations bilatérales. Acta Sci. Math. 23 (1962), $106-129$.

Received 14.02.1997; in revised form 12.01.1998 